\title{
RESEARCH HIGHLIGHT Nociceptor nerves set the stage for skin immunity
}

\author{
Felipe A. Pinho-Ribeiro ${ }^{1}$ and Isaac M. Chiu (D) \\ Cell Research (2019) 29:877-878; https://doi.org/10.1038/s41422-019-0240-x
}

\begin{abstract}
Nociceptor sensory neurons densely innervate the skin and detect potentially damaging stimuli. Cohen and colleagues show that nociceptor neurons drive local axonal reflexes that build anticipatory type 17 skin immune responses to protect the host against pathogens.
\end{abstract}

The skin is a barrier tissue whose loss of integrity must be promptly recognized and defended to prevent pathogen invasion. Pain is an unpleasant sensation that accompanies skin injury and is mediated by nociceptor somatosensory neurons. Nociceptors are specialized to detect damage-associated stimuli including mechanical injury, noxious heat, cold, and reactive chemicals. ${ }^{1}$ Nociceptors also have the ability to directly detect pathogens including Candida albicans, ${ }^{2,3}$ Streptococcus pyogenes, ${ }^{4}$ and Staphylococcus aureus. ${ }^{5}$ Nociceptors densely innervate the skin and have a characteristic wide coverage due to ramifications of their peripheral nerve branches. To produce adaptive responses, nociceptors initiate neural reflexes that integrate the activity of the receptor cell (in this case, a nociceptor neuron) to an effector cell to generate responses that protect from danger, which include pain-induced motor withdrawal and sympathetic reflexes.

Nociceptor activation triggers a local axonal reflex in the skin coupled with inflammation. This nerve-axon reflex arc initiates with action potential propagation from sensory nerve terminals, which upon reaching a branching point (orthodromic direction), can be transferred to other branches of the neuron (antidromic direction) adjacent to the stimulus. Antidromic signaling results in release of neuropeptides including calcitonin gene-related peptide (CGRP) and substance $P$ (SP) stored in vesicles at nerve terminals. CGRP and SP act on the vasculature and mast cells to mediate vasodilation, edema and inflammation. This "neurogenic inflammation" and flare response has been extensively documented $^{6,7}$, but its role in host-pathogen defense is not well understood.

In an elegant study, Cohen and colleagues ${ }^{8}$ demonstrate that $\mathrm{TRPV}^{+}$nociceptor neurons drive local axon reflexes in the skin to initiate protective type 17 immunity that anticipates pathogen invasion. Type 17 immunity (or Type 3 immunity) is characterized by activation of IL-17-producing cells including Th17 and $\gamma \delta$ $\mathrm{T}$ cells. In the skin, IL-17 induces recruitment of neutrophils to employ antimicrobial responses against extracellular pathogens like $C$. albicans and $S$. aureus. The authors recently showed that nociceptors mediated host defense against $C$. albicans by inducing type 17 immunity through CGRP. ${ }^{2}$ In the new study, ${ }^{8}$ the authors asked whether neurons could anticipate immunity prior to infection.

Cohen and colleagues used optogenetics to show that a type 17 immune response can be triggered purely by local activation of nociceptors in the absence of skin injury. Utilizing mice expressing ChR2 in TRPV1 ${ }^{+}$nociceptor neurons, the authors showed that repeated stimulation of ear skin nerves with blue light was sufficient to induce type 17 inflammation characterized by influx of $\gamma \delta-T$ cells, CD4a $\beta$ T cells, and neutrophils, and high levels of IL-17A, IL-23, IL-6, and TNF-a. This neurogenic immune response required CGRP release from vesicles, and effectively conferred protection to subsequent epicutaneous infection by skin pathogens $C$. albicans and $S$. aureus. Using an optical shield, the authors make an important observation: the effect of nerve stimulation was not limited to the stimulated area but also occurred at adjacent, unstimulated skin. Bupivacaine treatment (which inhibits sodium channels and action potentials) blocked increased blood flow at the adjacent area, providing evidence that this process was dependent on a nerve reflex arc. Epicutaneous infection also induced CGRP release and type 17 immunity in the uninfected, surrounding skin, which contributed to containing the spread of infection. This process, called anticipatory immunity, would allow a signal generated in a specific region to travel and generate protective immune reflexes at the yet unaffected surrounding area (Fig. 1).

Therefore, in an attempt to understand the neurobiology behind skin infection, the authors discovered a novel aspect of immunology triggered by the nervous system. Given the relative speed of neural reflexes (within seconds) compared to immune responses (minutes to hours), neuronal anticipation of immunity could represent a powerful early organismic response to potential danger. This capacity to predict injury suggests that nociceptors can shape immunity before injury or infection occurs. Neuronal control of immunity, while protective in host defense, has also been implicated in disease pathogenesis. The same neural reflex that promotes skin immunity against pathogens ${ }^{8}$ drives detrimental type 17 skin inflammation in a mouse model of psoriasislike disease. ${ }^{9}$ Pathogens can also exploit the influence of neurons on immune responses. ${ }^{4,5}$ S. pyogenes causes CGRP release during subcutaneous necrotizing invasion that blocks neutrophil influx and antimicrobial activity. ${ }^{4}$ To avoid immunopathology, neurons and immune cells also promote tissue-specific, counter-regulatory reactions. While CGRP drives type 17 immunity, ${ }^{2,8}$ it suppresses TNF production by macrophages through ICER-dependent signaling. ${ }^{10}$ Neuro-immune signaling may be influenced by the sensory neuronal subtype involved, the type of pathogen (bacteria or fungal or parasite strain), neuronal mediator (CGRP or SP), responding immune cell (innate or adaptive), and site of infection (epicutaneous, deep tissue, or subcutaneous). The study of Cohen and collaborators adds an important piece to the study of neuroimmune interactions and provides new insights into host defense and inflammation.

'Department of Immunology, Harvard Medical School, 77 Avenue Louis Pasteur, Boston, MA 02115, USA

Correspondence: Isaac M. Chiu (Isaac_chiu@hms.harvard.edu)

Published online: 16 October 2019 


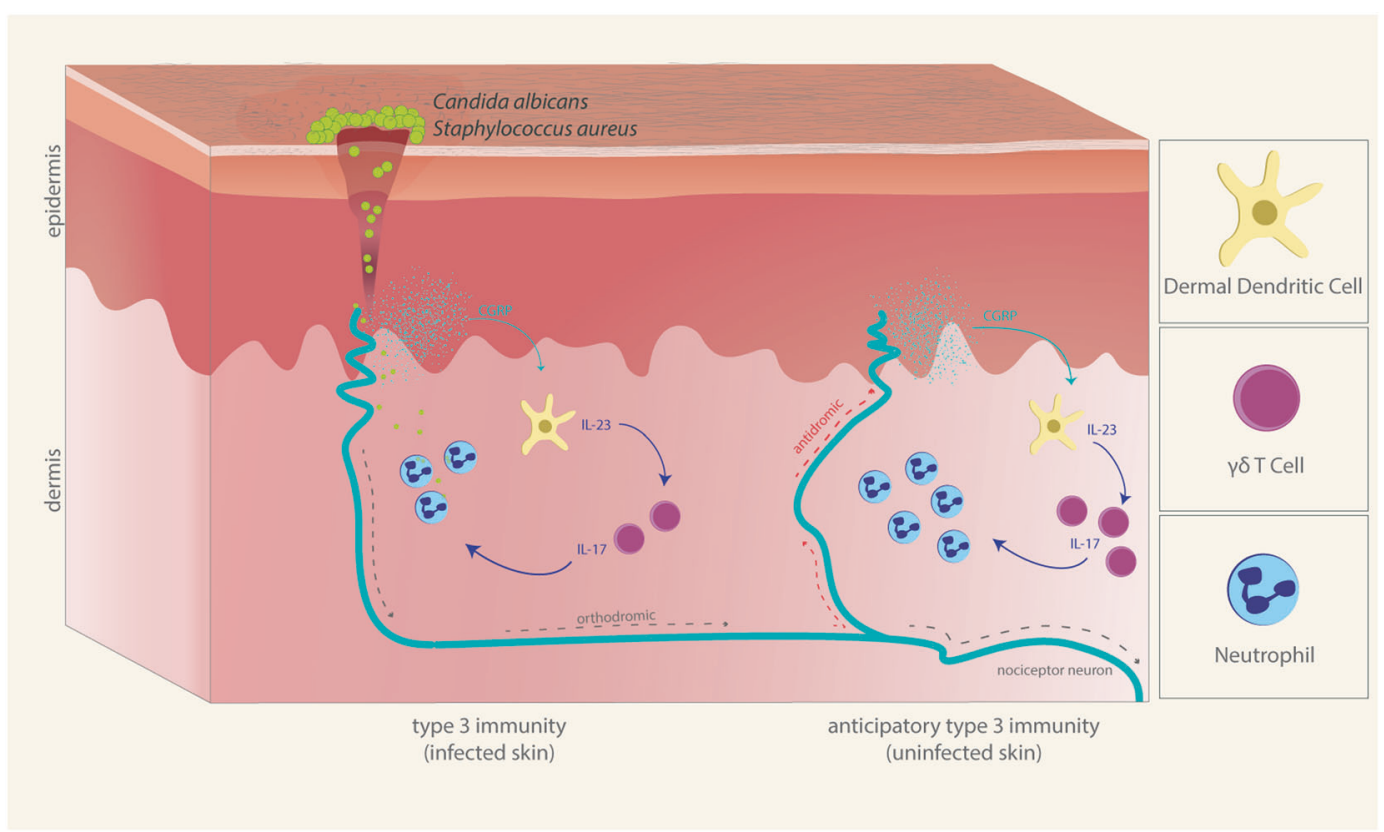

Fig. 1 Nociceptor neurons drive anticipatory type 17 immunity in the skin. Skin infection or other noxious stimuli stimulate TRPV1 ${ }^{+}$ nociceptor neurons to generate action potentials that travel orthodromically to the central nervous system and antidromically to adjacent nerve branches in an axonal reflex. These action potentials induce CGRP release from vesicles stored within nerve terminals at the site of infection and at neighboring sites of uninfected skin. CGRP acts on dermal dendritic cells to produce IL-23, which in turn induces $\gamma \delta$-T cells to produce IL-17 that mediates neutrophil recruitment to promote immunity against pathogens including $S$. aureus and C. albicans

\section{ADDITIONAL INFORMATION}

Competing interests: The authors declare no competing interests.

\section{REFERENCES}

1. Basbaum, A. I., Bautista, D. M., Scherrer, G. \& Julius, D. Cell 139, 267-284 (2009).

2. Kashem, S. W. et al. Immunity 43, 515-526 (2015).
3. Maruyama, K. et al. Cell Rep. 19, 2730-2742 (2017).

4. Pinho-Ribeiro, F. A. et al. Cell 173, 1083-1097 (2018).

5. Chiu, I. M. et al. Nature 501, 52-57 (2013).

6. Pinho-Ribeiro, F. A., Verri, W. A. Jr. \& Chiu, I. M. Trends Immunol. 38, 5-19 (2017).

7. Lewis, T. Br. Med. J. 1, 431-435 (1937).

8. Cohen, J. A. et al. Cell 178, 919-932 (2019).

9. Riol-Blanco, L. et al. Nature 510, 157-161 (2014).

10. Harzenetter, M. D. et al. J. Immunol. 179, 607-615 (2007). 